\title{
Artificial Intelligence Effectiveness and Impact within COVID-19
}

\author{
Ragad M Tawafak ${ }^{1 *}$, Ghaliya Alfarsi ${ }^{1}$, and Baidaa Hamza Khudayer ${ }^{1}$ \\ ${ }^{1}$ Department of Information Technology, AlBuraimi University College, Buraimi, Oman
}

\begin{abstract}
Artificial intelligence is one of the most important programs that are used in all sectors of society. Accordingly, artificial intelligence has become required to help combat Covid 19. In the field of education, artificial intelligence has become the ideal alternative to study and complementary to distance study. This research provides a summary of the types of artificial intelligence, its benefits, drawbacks, and the field of its uses. This paper will present the importance of using intelligence Artificial intelligence in the field of education specifically and its importance in expanding students' awareness in the case of distance study.
\end{abstract}

\section{Introduction}

Artificial intelligence is being used professionally to combat the COVID-19 pandemic [1] after the occurrence of infections and the complete closure all over the world. During the difficult period [2] of the pandemic, the most common solution has been the use of artificial intelligence. Artificial intelligence has invaded all different aspects of life. This study focuses largely on the applications of artificial intelligence in the educational aspect to simulate the new method of education within the period of Covid 19. [3, 4].

Artificial intelligence has been used in the field of learning with multi-dimensional moving images. Where imaging takes place, many different moving images appear to reflect the opportunity of smart devices or robots [5]. These robots help describe complex objects by drawing detailed maps of the mechanics of objects and how they are made. They made machines to help them deliver more within the processing plants. Fortunately, this idea has generally been successful, and individuals still use dedicated devices to achieve great speed and high cost $[6,7]$.

This research is divided into several units. It begins with an introduction and general review of research using artificial intelligence in education and in motion pictures to serve learners. Next, an explanation of the types of artificial intelligence in the areas of life. Then, the benefits of artificial intelligence and some of the qualities that are obvious as drawbacks in its uses. In the end, the research refers to the summary of the paper and the supported recommendations for the use of artificial intelligence in the field of education and distance learning.

\footnotetext{
* Corresponding author: raghad@buc.edu.om
} 


\section{What is the necessity of Artificial Intelligence and its features?}

In this recent period, the measure of knowledge produced by two people goes beyond the plurality of robots and smart applications that help in the speed of completion and accuracy of information. [8, 9, 10]. In gaming, far fewer people would see excellent checkers bosses, with over $500 \times 1018$, or 500 quintillions, of various possible moves [11]. Personal computers are incredibly adept at checking these algorithms to indicate a more direct identification. [12]. Privacy and Security: Licensors can determine the effectiveness and efficiency of security and privacy settings. Protection is based on the concept of "use". It is to give powers to the agency that prepares the program and to enable it to freely add to external parties working on development and assistance [13]. Mutual Suspicious "memory-free" subsystems are implemented easily and efficiently. The Protection Officer has the right to direct access to menus relating to controls, objects, and capabilities. Matters related to protection always remain unresolved until the issue of trust and intent becomes clear from the party dealing with it [14].

There are a lot of data that stimulate the desire for appropriate evaluation methods for machines capable of performing complex tasks $[4,5]$, including the measure of scientific progress in the field of study: the degree of research progress, the percentage of variation of copies in the same system, identifying obstacles to modification and switching, between the components of a system One or more AI systems with different tasks [10]. The goal is to determine the variables that take into account speed, accuracy, reliability, independence, the reasons deduced from the relationships, the degree of complexity of things, and other factors that affect the construction of the smart system [15].

In the modern period of technological development, educational methods focused on the ability to play smart games by students as an indicator of the development of systems. Dealing with a variety of smart tools and algorithms to achieve the conditions of the game. Hence simple performance A rating such as the achieved game score cannot describe the progress of AI per se [6]. Since the general assessment of the player's ability to control the data and change the various characteristics, we can conclude the degree of the player's selfcontrol.

\section{Features- The New Age Electricity}

Eliminate boring and cumbersome processes [15].

Absorbing large and compressed information [16].

It imitates human perception in the speed of discrimination and reactions, for example, thwarting catastrophic events, the accuracy of facial recognition, chatbots, environmental protection speed mechanisms, the development of technology for the use of daily life, and others [17].

\section{Advantages and Disadvantages of Artificial Intelligence}

The positive aspects of synthetic knowledge applications are enormous and could work for any specialist department $[18,19]$. What do you think that we see a number of them?

\subsection{Reducing human error}

The emergence of this term is because people make mistakes from time to time $[1,9]$. These errors are simple and comprehensible, and some are complex and linked to other systems that negatively affect several delicate systems. Conversely, computers do not make these errors 
if they are properly customized [2, 12, 20]. As the synthetic knowledge mechanism, it is done by using the alternatives taken from the recently accumulated data from real and reliable sources by applying the chosen order of the calculations. So, errors are automatically reduced, so the possibility of reaching accuracy with a detectable level of accuracy may be an opportunity [2, 12].

For example: When anticipating the use of AI, they need to reduce most human errors and human use as possible and replace them with sophisticated robots.

\subsection{Takes risks instead of humans}

Building and developing robots that are used specifically for rescue operations or to use in areas that are not safe for humans. For example: defusing the bomb, inspecting the deepest parts of the seas, drilling for coal and oil, analyzing and prior detection of infected people, especially in the Covid-19 period, and tending to be used successfully in any form of natural or man-made disasters [2, 3].

Example: Have you ever been exposed to the winds of an explosion at the Chernobyl atomic power plant in Ukraine? At that time, there were no artificial intelligence robots that could help us reduce the impact of radiation by controlling the stove ahead of time since any human who gets close to the middle dies very quickly [4]. In the end, they poured sand and boron from helicopters from a small interval [5]. Figure 1, shows a basic algorithm and an advanced one to find a smart solution.

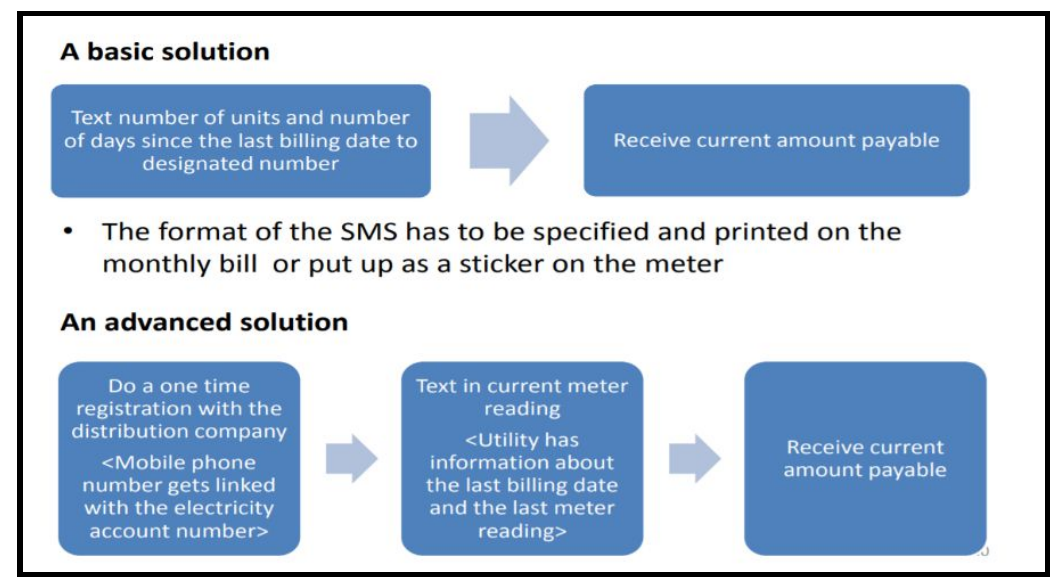

Fig. 1. Smart solution using AI algorithms

\subsection{Available $24 \times 7$}

The daily and normal work of the average person ranges between 4-6 hours a day, excluding rest periods. During the break, people work to motivate themselves and prove themselves against another working day. But in the case of using robots and artificial intelligence software, unlike people, we will make the machines work 24/7 without breaks and will not be stopped, making the work output double the original work if it was under human management $[2,6]$.

Model: Educational institutes and help centres are now providing solutions to many questions and issues that can be addressed for the successful use of AI through algorithms that discover frequently asked questions and hypothetical solutions for each case. Figure 2, shows the $24 \times 7$ camera working with AI development. 


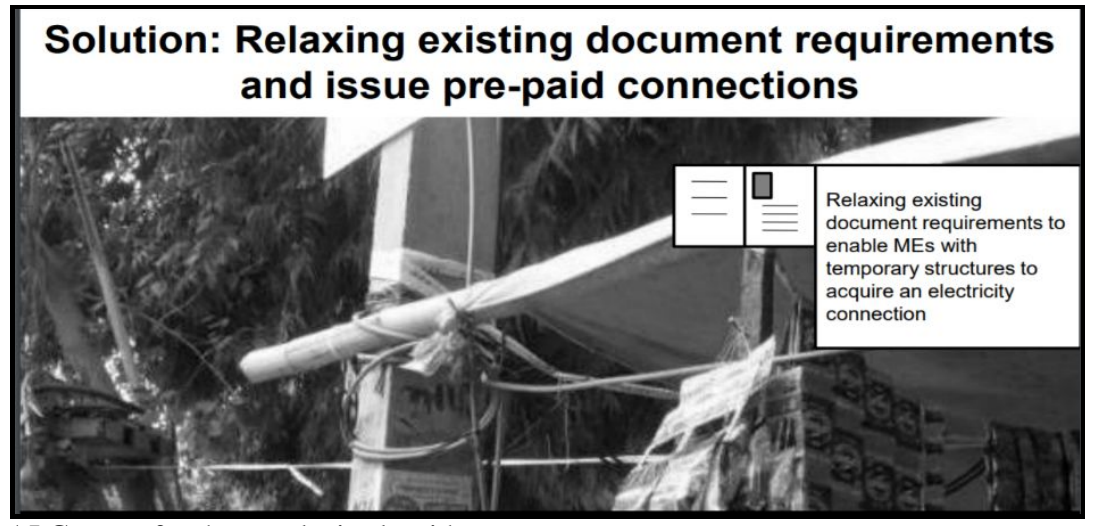

Fig. 2. AI Camera for data analysis algorithms

\subsection{Digital assistance}

Organizations and associations rely on ready-made AI-related software to interact with customers to reduce the need for human resources. Some chatbots were planned so that it would be difficult to verify our visit using a chatbot or personal software [7, 8, 9].

Form: We all understand that associations have a customer assistance group that requires clarifying customer questions and inquiries. Using artificial intelligence, associations can create a voice bot or chatbot that may help customers respond to each of their problems. We will see many associations that have already started using it on their various websites and apps [3].

\section{Disadvantages}

\subsection{High construction costs}

Due to the continuous renewal and updating in this field related to artificial intelligence, and the mechanism used to clarify the curricula in the case of distance learning, as is required especially in the medical field and the accompanying development of diagnostic software for Covid 19. Equipment and programming must be updated over time to meet the modern basic requirements. This process remains with many drawbacks, as the machines need to be repaired and maintained, which is very expensive. Their creation requires huge expenditures because they are amazing machines $[2,3,13]$.

\subsection{Make people lazy}

Excessive reliance on artificial intelligence reduces public awareness and users' desire to discover new aspects. Which makes the opportunity for developing the preserve of a few individuals, and this negatively affects the general perception and the main goal of making advanced software. People are often drawn to these innovations that may make people a problem in the future $[3,4]$.

\subsection{Unemployment}

Since AI replaces most of the monotonous tasks and various work with robots, the human impedance appears with less firmness making it capable of causing a significant problem 
within work processes. Each association hopes to replace the less qualified people with AI robots that may do work compared to greater productivity $[3,15]$.

\subsection{No emotions}

Of course, machines have greatly improved in terms of working efficiently, but they cannot replace the human bond that creates the group. They lack sensory and emotional perception, and devices cannot build a relationship with people which may be an essential asset when team management is involved $[12,16]$.

\section{Conclusion}

Computerized thinking, as well as innovation, is one aspect of existence that continually interests us and shocks us with ideas, topics, developments, new elements, etc. In the current year 2020, life has witnessed rapid and dramatic changes during the great spread of the COVID-19 epidemic, which was made by humans with artificial intelligence to limit the spread of the virus between countries. Artificial intelligence has used smart robots as an anxiety complex solution to reduce infection and as a magic solution to control world lives differently through online controls. Anyway, hidden tasks and so is the progress in modern organizations.

Towards the top, we scrolled through this examination through definitions of AI, brief history, uses of AI in public, and uses of AI. Uses of AI in the military, morals of AI, and three guidelines of mechanical autonomy. It is not the finish of AI, there's an entire other world to originate from it, who recognizes what the AI can accomplish for us presently, perhaps it'll be a whole society of robots.

\section{References}

1. Naudé, Wim. "Artificial Intelligence against COVID-19: An early review." (2020).

2. Shi, Feng, Jun Wang, Jun Shi, Ziyan Wu, Qian Wang, Zhenyu Tang, Kelei He, Yinghuan Shi, and Dinggang Shen. "Review of artificial intelligence techniques in imaging data acquisition, segmentation, and diagnosis for COVID-19." IEEE reviews in biomedical engineering 14 (2020): 4-15.

3. Tawafak, Ragad M., Awanis M. Romli, and Maryam Juma Alsinani. "Student assessment feedback effectiveness model for enhancing teaching method and developing academic performance." International Journal of Information and Communication Technology Education (IJICTE) 15, no. 3 (2019): 75-88.

4. Mohamadou, Youssoufa, Aminou Halidou, and Pascalin Tiam Kapen. "A review of mathematical modelling, artificial intelligence and datasets used in the study, prediction and management of COVID-19." Applied Intelligence 50, no. 11 (2020): 3913-3925.

5. Tatnall, Arthur. "Correction to Editorial for EAIT issue 2, 2020." Education and Information Technologies 25, no. 6 (2020): 5901-5910.

6. Malik, Sohail, Mostafa Al-Emran, Roy Mathew, Ragad Tawafak, and Ghaliya AlFarsi. "Comparison of E-Learning, M-Learning and Game-based Learning in Programming Education-A Gendered Analysis." International Journal of Emerging Technologies in Learning (iJET) 15, no. 15 (2020): 133-146.

7. Tawafak, Ragad M., Ghaliya Alfarsi, Awanis Romli, Jasiya Jabbar, Sohail Iqbal Malik, and Abir Alsideiri. "A Review Paper on Student-Graduate Advisory Expert system." 
In 2020 International Conference on Computing and Information Technology (ICCIT1441), pp. 1-5. IEEE, 2020.

8. Tawafak, Ragad, Ghaliya AlFarsi, Jasiya Jabbar, Sohail Iqbal Malik, Roy Mathew, Abir AlSidiri, Mohanaad Shakir, and Awanis Romli. "Impact of Technologies During COVID-19 Pandemic for Improving Behavior Intention to Use E-learning." (2021): 184-198.

9. Tawafak, Ragad M., Sohail Iqbal Malik, and Ghaliya Alfarsi. "Impact of Technologies During the COVID-19 Pandemic for Improving Behavioral Intention to Use ELearning." International Journal of Information and Communication Technology Education (IJICTE) 17, no. 3 (2021): 137-150.

10. Iqbal Malik, Sohail, Roy Mathew, Ragad M. Tawafak, and Ghaliya Alfarsi. "A webbased model to enhance algorithmic thinking for novice programmers." E-Learning and Digital Media 18, no. 6 (2021): 616-633.

11. Malik, Sohail Iqbal, Roy Mathew, Abir Al-Sideiri, Jasiya Jabbar, Rim Al-Nuaimi, and Ragad M. Tawafak. "Enhancing problem-solving skills of novice programmers in an introductory programming course." Computer Applications in Engineering Education (2021).

12. Tawafak, Ragad M., Ghaliya Alfarsi, and Jasiya Jabbar. "Innovative Smart Phone Learning System for Graphical Systems within COVID-19 Pandemic." Contemporary Educational Technology 13, no. 3 (2021): ep306.

13. Tawafak, Ragad, Sohail Malik, Roy Mathew, Mohammed Ashfaque, Jasiya Jabbar, Maryam AlNuaimi, Abdalla ElDow, and Ghaliya Alfarsi. "A Combined Model for Continuous Intention to Use E-Learning System." (2021): 113-129.

14. Malik, Sohail Iqbal, Ragad M. Tawafak, and Mohanaad Shakir. "Aligning and Assessing Teaching Approaches With SOLO Taxonomy in a Computer Programming Course." International Journal of Information and Communication Technology Education (IJICTE) 17, no. 4 (2021): 1-15.

15. Nayal, Kirti, Rakesh Raut, Pragati Priyadarshinee, Balkrishna Eknath Narkhede, Yigit Kazancoglu, and Vaibhav Narwane. "Exploring the role of artificial intelligence in managing agricultural supply chain risk to counter the impacts of the COVID-19 pandemic." The International Journal of Logistics Management (2021).

16. Nogueira, Raul G., Jason M. Davies, Rishi Gupta, Ameer E. Hassan, Thomas Devlin, Diogo C. Haussen, Mahmoud H. Mohammaden et al. "Epidemiological surveillance of the impact of the COVID-19 pandemic on stroke care using artificial intelligence." Stroke 52, no. 5 (2021): 1682-1690.

17. Sharifi, Abbas, Mohsen Ahmadi, and Ali Ala. "The impact of artificial intelligence and digital style on industry and energy post-COVID-19 pandemic." Environmental Science and Pollution Research 28, no. 34 (2021): 46964-46984.

18. Sharifi, Abbas, Mohsen Ahmadi, and Ali Ala. "The impact of artificial intelligence and digital style on industry and energy post-COVID-19 pandemic." Environmental Science and Pollution Research 28, no. 34 (2021): 46964-46984.

19. Jabbar, Jasiya, Sohail Iqbal Malik, Ghaliya AlFarsi, and Ragad M. Tawafak. "The Impact of WhatsApp on Employees in Higher Education." In Recent Advances in Intelligent Systems and Smart Applications, pp. 639-651. Springer, Cham, 2021.

20. Al Farsi, Ghaliya, Azmi bin Mohd Yusof, Awanis Romli, Ragad M. Tawafak, Sohail Iqbal Malik, Jasiya Jabbar, and Mohd Ezanee Bin Rsuli. "A Review of Virtual Reality Applications in an Educational Domain." International Journal of Interactive Mobile Technologies 15, no. 22 (2021). 